\title{
Compartmentation of Metabolites in Regulating Epigenomes of Cancer
}

\author{
Zhiqiang Zhao, ${ }^{1} \mathrm{Li}$ Wang ${ }^{1,2}$ and $\mathrm{Li}-\mathrm{jun} \mathrm{Di}{ }^{1}$
}

${ }^{1}$ Faculty of Health Sciences; and ${ }^{2}$ Metabolomics Core, Faculty of Health Sciences, University of Macau, Macau SAR, China

\begin{abstract}
Covalent modifications of DNA and histones are important epigenetic events and the genomewide reshaping of epigenetic markers is common in cancer. Epigenetic markers are produced by enzymatic reactions, and some of these reactions require the presence of metabolites, specifically Epigenetic Enzyme Required Metabolites (EERMs), as cofactors. Recent studies found that the abundance of these EERMs correlates with epigenetic enzyme activities. Also, the subcellular compartmentation, especially the nuclear localization of these EERMs, may play a role in regulating the activities of epigenetic enzymes. Moreover, genespecific recruitment of enzymes that produce the EERMs in the proximity of the epigenetic modification events accompanying the regulation of gene expression, were proposed. Therefore, it is important to summarize findings of EERMs in regulating epigenetic modifications at both the DNA and histone levels, and to understand how EERMs contribute to cancer development by addressing their global versus local distribution.
\end{abstract}

Online address: http://www.molmed.org

doi: $10.2119 / \mathrm{molmed} .2016 .00051$

\section{INTRODUCTION}

Epigenetics refers to modifications in gene expression caused by heritable, but potentially reversible, alterations in chromatin structure and/or DNA methylation without changes in DNA sequence (1). Thus epigenetic modifications have the ability to directly regulate gene expression and the information "coded" by modifications could be passed to progeny cells and act as a memory to form a consistent pattern of gene expression (2). Interruption of epigenetic modifications frequently associates with diseases. Epigenetic modifications only occur in the nucleus and the targets include both DNA and histones. Known epigenetic modifications include DNA methylation, histone modifications such as acetylation, ubiquitination, methylation, phosphorylation (3), sumoylation (4), crotonylation (5), glycosylation (O-GlcNAc, or O-linked $\mathrm{N}$-acetylglucosamine) (6) and biotinylation $(7)$ and others $(8,9)$. Generally, one step or multiple steps of enzymatic reactions are required to generate each epigenetic modification and, in many cases, the EERMs that form the modifications have to be present (Figure 1). The occurrences of epigenetic modifications are potentially subjected to two regulatory mechanisms: recruitment of the enzyme itself and/or the presence of the EERMs. To date, the recruitment of the respective enzyme in generating the epigenetic modification has been characterized with much more detail by far, but research realizing that the presence of EERMs may also have some regulatory roles based on their compartmentalized distribution is still in the beginning stages.
Address correspondence to Li-jun Di, Faculty of Health Sciences, University of Macau, Macau SAR, China. Phone: 853-8822-4498; Fax: 853-8822-2314; E-mail: lijundi@umac.mo. Submitted February 22, 2016; Accepted for publication April 14, 2016; Published Online (www.molmed.org) April 18, 2016.$$
\text { ฟทำ }
$$$$
\text { Feinstein Institute }
$$$$
\text { for Medical Research }
$$$$
\text { Northwell Health }
$$

Among the epigenetic modifications, ubiquitination and sumoylation require ubiquitin and sumo rather than metabolites as cofactors and will not be discussed here. For histone phosphorylation, ATP is the EERM for most of the histone kinases. Since ATP is mainly synthesized within mitochondria, diffusion is the dominant way for ATP to be present in the nucleus, and as there is no locally enriched ATP in the nucleus at all, histone phosphorylation may not be influenced by ATP and may be regulated solely by the local recruitment of kinases. Crotonylation of histones has been discovered very recently and the importance of this epigenetic modification in cancer biology is still under investigation. Biotinylation of histones or other proteins also occurs quite abundantly in mammalian cells, but the importance of biotinylation in cancer is not known yet. Therefore, we will focus mainly on the EERMs required for methylation/demethylation as well as on acetylation/ deacetylation.

EERMs, in generating epigenetic modifications, create a potential regulatory mechanism through which the epigenetic information can be regulated 


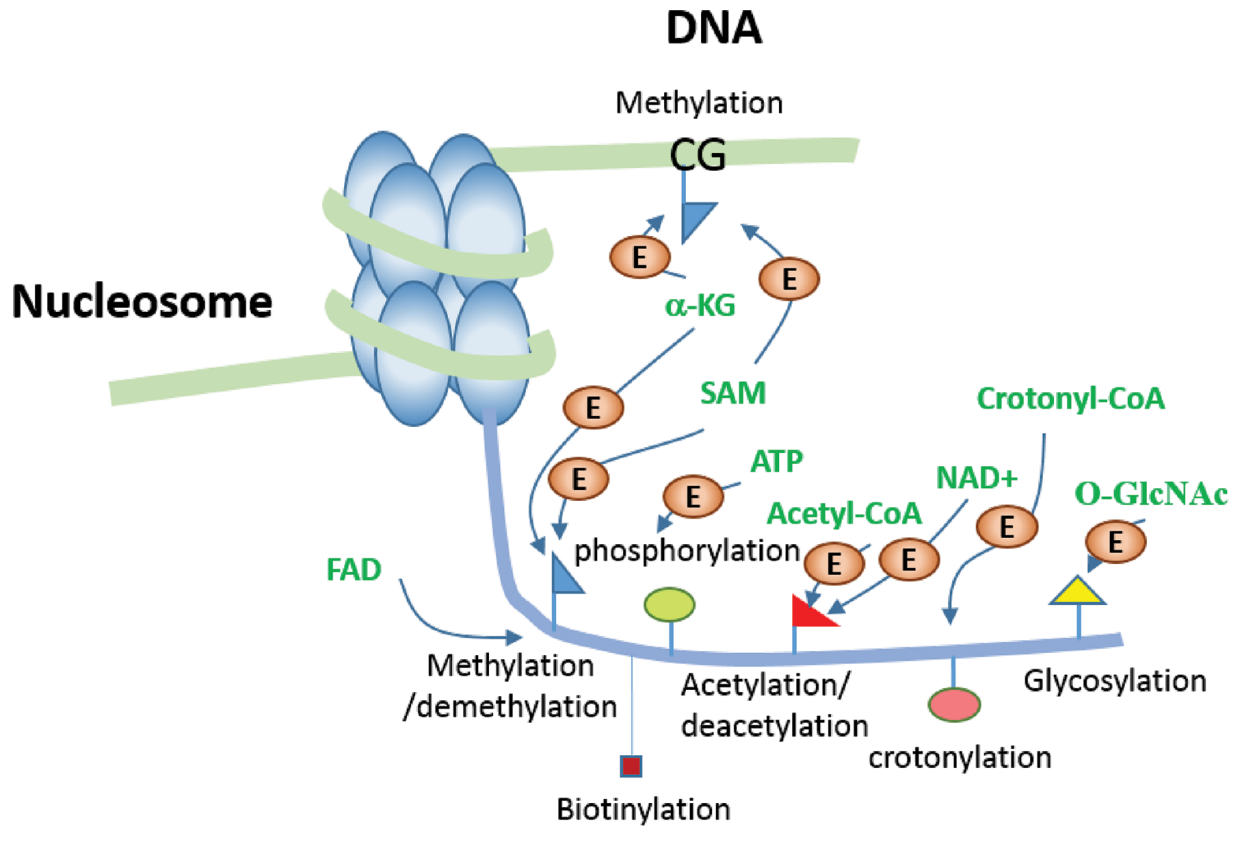

Histone tail

Figure 1. Epigenetic markers and EERMs. Schematic representation of epigenetic markers on histone tails and DNA strand. Various enzymes (E) are responsible for the generation of epigenetic modification including DNA methylation/demethylation, histone acetylation/deacetylation, histone methylation/demethylation, histone biotinylation, crotonylation, phosphorylation and glycosylation with the presence of EERMs (dark green) including SAM, $\alpha-K G, F A D$, acetyl-CoA, NAD ${ }^{+}$, crotonyl-CoA and O-GICNAC

via metabolic pathways. Abnormal cellular metabolism is considered one of the hallmarks of cancer (10). Along with these irregular metabolic pathways are global changes in abundance of, as well as compartmentation of, metabolites including EERMs (11-14). A well-recognized hypothesis to explain the correlation between EERMs and cancer is that epigenetic modification enzymes are able to conceive the metabolic status of each individual EERM and interpret the cellular metabolic status as chromatin modification pattern that ultimately determines the gene expression profile (13,15-19).

EERMs are intermediates of metabolic pathways and, therefore, their production is subjected to the presence and activity of respective enzymatic reaction. Current knowledge suggests that EERM production mainly occurs in the cytoplasm and diffusion of EERMs into the nucleus is the dominant way for EERMs to be involved in epigenetic modifications. But more and more, metabolic enzymes are identified within the nucleus with important functions in regulating gene expression by serving as cofactors of transcriptional regulatory complexes $(18,19,20-22,23-28)$. Some of these factors actually have the enzymatic activities to produce EERMs locally along with other nuclear events such as gene transcription and DNA replication (18,19,23-26).

Distinct to the universal change of EERMs, the local enrichment of the enzymes that generate EERMs may represent a novel regulatory mechanism that operates independently of or dependent on global changes of the respective EERMs. In this review, we summarize the recent findings regarding the role of metabolic pathways in influencing the abundance of many EERMs in different compartments within the cell and analyze how EERMs regulate both global and local epigenetic events within the nucleus and how EERMs regulate cellular processes that may be causative to cancer.

\section{S-ADENOSYL-METHIONINE AND METHYLATION}

Both DNA methylation and histone methylation need S-adenosyl-methionine (SAM) as the methyl group donor.

Aberrant DNA methylation and histone methylation are dominant markers of cancer and DNA-methylation-associated tumor suppressor gene inactivation is a common feature of cancer (29-32). DNA methylation in particular is a reversible epigenetic modification dynamically maintained throughout the cell cycle and is regulated by the DNA methyltransferases DNMT1 3a and 3b, which transfer methyl groups from SAM to cytosine (33). Distinct to DNA methylation, histone methylation is much more complicated and almost all the histone variants have arginine and/or lysine residues methylated. But $\mathrm{H} 3$ and $\mathrm{H} 4$ are the predominant targets of methylation by histone methyltransferases (HMTs), which transfer the methyl group from SAM to lysine or arginine. In most of the cases, hypermethylation of DNA associates with decreased gene expression owing to the recognition of the methylated cytosine by methylCpG-binding proteins (MBPs), which potentially shield the nucleotides from being bound by transcriptional activators and from recruitment of other chromatin-silencing factors $(29,34)$. But methylation of histones may generate different outcomes depending on the location of the methylated residue. For instance, methylation of H3K9, H3K27 and H4K20 usually results in transcriptional repression, while methylation of H3K4, H3K36 and H3K79 usually activates gene transcription (35-37). Histone methylation is even more complicated in that each residue can acquire a different number of methyl groups that may result in a distinct chromatin status, adding more complexity to SAM-mediated histone modifications. 
There are many methyl group donors within mammalian cells, and SAM is the dominant one for epigenetic modifications (38). SAM is synthesized mainly in the cytoplasm and is biosynthesized through transferring the adenosyl group of ATP to methionine by methionine adenosyltransferases (MATs) (39). Methionine is one of the essential amino acids for mammalian cells and has to be supplied through diet (40), rendering methionine, as well as SAM, the ability to mediate the signal from diet to intracellular epigenetic modifications of chromatin. The methionine is recovered from methyl-transfer reaction mediated by SAM in the form of S-adenosylhomocysteine (SAH), which will be converted to homocysteine (Hcy) and further to methionine. Hcy-to-methionine conversion requires another methyl donor, 5-methyl-tetrahydrofolate (5-methyl-THF), and this reaction needs vitamin B12 (41). 5-meth$\mathrm{yl}$-THF is mainly produced through the folate cycle, and the folate cycle serves as the mediator between the one carbon metabolism and the methionine cycle (42). Increasing the folate cycle intermediates THF and can be critical to boost up the level of SAM and SAM-related methylation (43).

A higher level of SAM in cancer cells is possibly determined by at least two factors. Both factors are based on the fact that cancer cells have a higher rate of glycolysis owing to the Warburg effect $(10,11,44)$. One factor is that there are surpluses of both ATP production and 5-methyl-THF production owing to the enhancement of glycolysis in cancer cells. Therefore the rate-limiting step of SAM formation is actually determined by the rate of the methionine cycle. Previous studies showed that cancer cells might have a higher amount of acquirement for methionine $(45,46)$, suggesting that increasing methionine absorption or accelerating the methionine cycle can boost the supply of SAM. The second factor is that cancer cells have a higher rate of one carbon metabolism (47) as well as a higher amount of folate-cycle intermediates (42). For instance, 5-methyl-THF can be generated during serineto-glycine conversion (42) and serine production can be a major output of 3-PG in some cancer cells (47). A very recent study also pointed out the possibility that serine production may contribute mainly to purine synthesis, especially the de novo synthesis of ATP that is essential for methionine-to-SAM conversion (48). Moreover, glycine decarboxylation also contributes to the abundance of 5-methylTHF in cancer cells (49).

A greater abundance of SAM in cancer cells, however, is not always correlated with global hypermethylation of genome DNA. Instead, the hypomethylated genome of cancer cells is much more common, which may be causative to tumorigenesis owing to decreased genome stability. However, hypermethylation of some tumor suppressor genes is a very communal mechanism for tumorigenesis (50). Such inconsistency suggests that SAM abundance may not be the rate-limiting factor dictating global DNA methylation. The gene-specific recruitment of methyl-transferases, however, may be the major reason for local enrichment of DNA methylation, along with a global background of hypomethylation (32). Another possibility can be the gene-specific recruitment of DNA demethylases (see following section $\alpha-K G$ AND DNA DEMETHYLATION). Other studies also suggested the local recruitment of SAM synthase $(19,25,26,51)$. For instance, a multi-protein complex SAM-integrating transcription repression (SAMIT) was proposed that includes MATs to produce SAM locally to repress gene expression through DNA methylation $(25,26)$. Although these studies only reported the recruitment of SAMIT to MafK-repressed genes, the potential of local recruitment of MATs to other gene loci by different transcription factors cannot be excluded. In fact, the interacting proteins of MATs are quite diversified and many of them are known cofactors of gene-expression regulation.

Even though histone methylation at several residues were studied as correlative factors of cancer, the relationship between SAM level and histone methylation has not yet been established. Only one recent study observed that H3K4 methylation is subjected to regulation by increased SAM and all the other residues, including H3K9, H3K27, H3K36 and H3K79, were not affected $(17,52)$. But this study was limited to the global change of SAM, and if there is any local enrichment of SAM production along with histone methylation it is still not known.

\section{$\alpha-K G$ AND DNA DEMETHYLATION}

Both DNA and histone methylation are dynamically regulated through demethylases and all the known demethylases require the presence of EERMs. Interestingly, some DNA demethylases and histone demethylases share the same EERM, $\alpha$-ketoglutarate $(\alpha-K G)$. Intracellular $\alpha-K G$ is derived mainly from the glycolysis pathway via decarboxylation of pyruvate and the TCA anaplerosis pathway via glutaminolysis (53). Simultaneously, $\alpha-\mathrm{KG}$ is also converted to citrate, catalyzed by iso-citrate dehydrogenases (IDHs), and to succinate, catalyzed by $\alpha-K G$ dehydrogenase $(53,54)$. Thus, intracellular enrichment of $\alpha-K G$ is very dynamic and subject to the cellular fluctuation of metabolic activities.

Conversion of $\alpha-\mathrm{KG}$ to succinate is coupled to the demethylation reactions catalyzed by demethylases which belong to dioxygenases, including the DNA demethylases Ten-Eleven Translocation family proteins (TET) and histone demethylases Jumonji domain-containing proteins (JMJD) $(55,56,57,58,59)$. The dynamic change of $\alpha-K G$ enrichment often is correlated with the activities of these demethylases. However the change of $\alpha-K G$ enrichment is not consistent in different studies. The best-characterized factor that constantly drives down $\alpha-K G$ is IDH mutation that converts $\alpha-K G$ to its hydroxyl analog, and leukemia with IDH mutation has an impairment of TET function and the global DNA hypermethylation $(60,61)$. On other cases that are irrelevant to IDH mutations, 
increased $\alpha-K G$ was observed, which leads to a pan-cancer genome reduction of DNA methylation, especially at the highly repetitive sequences and lowdensity CpG regions $(44,54,62,63,64)$. But a recent study actually suggested that hypoxia or lactate acidosis, both conditions commonly associated with almost all types of solid tumor growth, decrease $\alpha-K G$, and at least one gene, ETV4, was identified as regulated by $\alpha-K G$ epigenetically (65). The inconsistency in $\alpha-K G$ among tumors may be caused by the dynamic nature of $\alpha-K G$, which is difficult to measure.

As we discussed above, the hypomethylated genome in cancer cells still has hypermethylated local chromatin regions that frequently contain tumor suppressor genes. Diverting $\alpha-K G$ production enzymes from those tumor suppressor genes may be one of the potential mechanisms (50). However, the dynamic fluctuation of intracellular $\alpha-K G$ prevents the further characterization of its involvement in gene-locus-specific regulation of expression. Furthermore, none of the known enzymes that either produce or exhaust $\alpha-K G$ has been demonstrated to be involved in nuclear activities so far.

\section{HISTONE DEMETHYLATION AND COFACTORS}

The working mechanism of $\alpha-K G$ dependent histone demethylases is relatively simple compared to DNA demethylases. The methyl group of histone residuals is hydroxylated and released from the histone when $\alpha-K G$ is decarboxylated to succinate (59). Multiple members of this group of histone demethylases were identified as belonging to the evolutionarily conserved family of JMJD proteins that share the highly conserved JmjC domain (59).

A global effect of $\alpha-K G$ on histone demethylases has already been demonstrated. For instance, fumarate and succinate were found to inhibit $\alpha-K G$-mediated demethylation reactions, because fumarate and succinate share structural similarity with $\alpha-K G$ except that the keto group is absent
(66). Therefore, mutation of fumarate hydratase $(\mathrm{FH})$ and succinate dehydrogenase $(\mathrm{SDH})$, the enzymes catalyzing the conversion of fumarate to malate and succinate to fumarate, respectively, were found to be associated with accumulation of fumarate and succinate in the growing tumors. Consequently, some genes were silenced owing to the defect in $\alpha-K G$-dependent demethylation (66). Further evidences supporting the role of $\alpha-K G$ in global histone methylation regulation came from the study on 2-hydroxylglutarate (2-HG), a byproduct of mutant IDH. Mutation of IDH1 and IDH2 in cancer, in particular in brain tumors and leukemia, results in the accumulation of 2-HG, which also competitively represses the $\alpha-K G$-mediated demethylation $(14,60)$. In some recent studies, enrichment of 2-HG in tumor cells can also be mutant-IDH independent (67), but the effect of 2-HG in inhibiting $\alpha-K G$-dependent demethylation is still the same. In fact, L-enantiomer of 2-HG (L-2HG) is also identified as an EERM and regulates histone demethylation in kidney cancer (68).

There is another group of histone demethylases known as LSD1 that requires Flavin adenine dinucleotide (FAD) as the substrate $(69,70)$. Excessive expression of LSD1 has been associated with prostate cancer, undifferentiated malignant neuroblastoma, estrogen-receptor-negative breast cancer, lung and colorectal carcinoma and bladder cancer, suggesting a gain of function can be important for LSD1 to be oncogenic (71-74). Giving that FAD is an EERM for LSD1 function, the importance of this essential metabolite has been studied for its role in mediating LSD1 function. FAD is synthesized from riboflavin via the riboflavin kinase that recruits ATP as the substrate. ATP levels affect FAD availability and consequently influence the activity of LSD1 (75). Riboflavin is also known as vitamin B2, indicating that FAD enrichment relies on a dietary supply of riboflavin. Recent reports found that the level of FAD has an impact on the activity of LSD1. For instance, riboflavin deficiency in Jurkat cells was found to influence the activity of lysine (K)-specific demethylase 1A (LSD1) and to result in a global increase of H3K4 mono- or demethylation (76). Similar results were also identified in human hepatocarcinoma cells by the same group (77). In adipocytes, the availability of FAD was also found to be critical to LSD1 activity in regulating the genes that contribute to cellular energy expenditure (78). It is of note that the target genes affected by loss-of-function of LSD1 in different tissues are not the same, suggesting that LSD1 has a specialized regulatory spectrum in different types of cells. A recent study demonstrated that FAD may be synthesized locally within the nucleus because both nuclear enrichment of FAD and its synthase were detected (79), but whether the FAD synthase forms a complex with gene expression regulation factors such as LSD1 is still unknown.

\section{ACETYL-COA AND HISTONE ACETYLATION}

Histone acetylation is dynamically regulated by acetyl-transferases (HATs) and deacetylases (HDACs). HATs can be classified into several different families based on sequence homology and functions. The residues that are targeted by individual HATs are diversified and thoroughly reviewed by Marmorstein et al. (80). However, a common feature of most of the HATs is their dependence on acetyl-CoA as a major acetyl donor (81).

Acetyl-CoA synthesis is compartmented into mitochondria and cytosol because the mitochondria membrane is not permeable by acetyl-CoA (24), but the nuclear membrane is permeable by acetyl-CoA and there is no evidence to demonstrate the nuclear enrichment of acetyl-CoA. In mitochondria, pyruvate can be catalyzed by pyruvate decarboxylase complex (PDC) to produce acetyl-CoA that is used as a substrate to generate citrate catalyzed by the citrate synthase. Most of the mammalian cells have this critical step to start a cycle of reactions to release the energy hidden 
within the $\mathrm{C}-\mathrm{C}$ bond of acetyl-CoA. The acetyl-CoA in mitochondria has no effect on the histone acetylation. For the same reason, acetyl-CoA generated from fatty acid oxidation (reviewed by Carracedo et al. in 82) is mainly constrained within mitochondria, which may not be available to serve as the substrate for histone acetylation (24). However, the conversion from pyruvate to acetyl-CoA may also occur in cytosol because cytoplasmic PDC was recently identified (83). In this report, the correlation between the amount of PDC and histone acetylation was observed, suggesting the production of acetyl-CoA outside of mitochondria is important for histone acetylation. Moreover, this study indeed demonstrated that acetyl-CoA could be generated locally within the nucleus (83). However, no PDC interaction with nuclear proteins, especially the transcription factors, has been identified yet. Another independent pathway to generate acetyl-CoA outside of mitochondria is through ATP-citrate ligase (ACL) $(23,24)$. Shuttle of citrate outside of mitochondria to cytosol enables the ACL-mediated synthesis of cytoplasmic acetyl-CoA. The importance of ACL in producing acetyl-CoA and its subsequent usage as substrate in histone acetylation were recently demonstrated in highly proliferating cells (24), but the evidence to show the nuclear production of acetyl-CoA accompanying gene regulation is not available.

Cancer cells are well known for having higher rates of both glucose and glutamine consumption, which significantly boost the acetyl-CoA level and increase the histone acetylation level globally (84). A similar situation was also observed in highly proliferating ES cells, where, upon differentiation of ES cells, the acetyl-CoA is consumed through the energetic pathway and the histone acetylation is reduced (85). Recent studies in cancer cells further extended the significance of acetyl-CoA to cancer growth by addressing the acetyl-CoA synthesis through direct ligation between acetate and $\mathrm{CoA}$ relying on ATP (86-88). According to the interpretation of Comerford et al, the dependence of cancer cells on this type of acetyl-CoA synthesis reflects the critical need of cancer cells to reuse the acetate that may be released from histone deacetylation. If that is the case, this fast cycle of acetyl group may help to maintain a high level of acetylated histone, which is crucial to the survival of cancer cells. Moreover, acetate may be a more easily accessible substrate for some types of cancers and promote tumor growth as described $(84,86-88)$.

\section{HISTONE DEACETYLATION}

The reverse reaction of histone acetylation is histone deacetylation, which is mainly catalyzed by at least three families of histone deacetylases (HDACs) including HDAC type I and HDAC type II. Both HDAC I and II require no extra substrate for their deacetylation activities. In addition to HDAC I and II, a third family of HDACs was also identified, better known as the Sirtuin (SIRT) family of proteins, requiring $\mathrm{NAD}^{+}$as the cofactor. There are two types of enzyme activities associate with Sirtuin family members. One is the HDAC activity and the other is the ADP-ribosylase activity. Only SIRT1, 2, 6 and 7 are applicable in histone deacetylation because the rest of the Sirtuins either have no deacetylase activity or are restricted in mitochondria (89). As we described, acetylation of histones loosens the chromatin structure and generally associates with gene activation. So deacetylation of histones by Sirtuins in general will shut down the gene expression, no matter which residue is modified by Sirtuins.

Biosynthesis of $\mathrm{NAD}^{+}$starts with the essential amino acid tryptophan, which is taken up from the diet. Tryptophan goes through a series of enzymatic conversions to form quinolinic acid. Then quinolinate phosphoribosyltransferase catalyzes the formation of nicotinic acid mononucleotide (NAMN) by condensing quinolinic acid with 5-phospho- $\alpha$-D-ribose 1-diphosphate. NAMN is converted to
NA adenine dinucleotide (NAAD) by the enzyme nicotinamide mononucleotide (NMN) adenylyltransferase (NMNAT), then $\mathrm{NAD}^{+}$can be finally produced by $\mathrm{NAD}^{+}$synthase. However, the major synthesis pathway of $\mathrm{NAD}^{+}$in mammalian cells is the salvage pathway that starts from precursors such as nicotinic acid (NA) or nicotinamide (NAM).

NA can be converted to NAMN just as quinolinic acid can, and the subsequent enzymatic reactions are also the same as the de novo $\mathrm{NAD}^{+}$synthetic pathway. NAM, however, is converted to NMN, and NMN can be converted to $\mathrm{NAD}^{+}$by NMNAT consuming one ATP.

NAM and NA are known as vitamin B3, and the supply of vitamin B3 in mammalian cells is mainly through food intake. So far, there is no obvious evidence connecting the surplus NAM and/ or NA to cancer risk. Therefore, if NAM and/or NA have any role in cancer, the cancer may be caused by abnormal NAM and/or NA subcellular distribution. For instance, at least several enzymes in $\mathrm{NAD}^{+}$synthesis pathways are known to have multiple family members locating to different cell compartments (90). In the nucleus, $\mathrm{NAD}^{+}$is synthesized mainly through the salvage pathway, and the enzyme NMNAT-1, which catalyzes the NMN to NAD ${ }^{+}$, has an NLS domain, suggesting the local synthesis of $\mathrm{NAD}^{+}$ (91). Moreover, NMNAT-1 was found to form a complex with nuclear transcriptional factors such as MSX1 in large-scale protein-protein interaction studies, although the interaction still needs further validation and the functional significance is also not yet known (92). Another example is nuclear lactate dehydrogenase (nLDH), which was recently identified to catalyze the nuclear production of pyruvate and $\mathrm{NAD}^{+}$and which influences SirT1 should be SIRT1 activity and histone acetylation (93). NAD ${ }^{+}$also influences PARP family proteins. Both Sirtuin and PARP were shown to be specific gene transcription regulators by enzymatically modifying other factors using $\mathrm{NAD}^{+}$as the substrate within the nucleus $(94,95)$. 
The reduced $\mathrm{NAD}^{+}$is NADH that is not required by Sirtuin family proteins. However, NADH is able to mediate the dimerization of $\mathrm{CtBP}$ corepressors. $\mathrm{NADH}$ is unevenly distributed between the cytoplasm and nucleus based on observations using two-photon microscopy, fluorescence-lifetime image microscopy and genetically encoded fluorescent biosensors (96-99). The estimated enrichment of NADH in the nucleus is around $130 \mathrm{nmol} / \mathrm{L}$, which is well within the range required by $\mathrm{CtBP}$ (97). CtBP represses transcription through recruiting various histone-modifying enzymes such as HDACs, histone H3 lysine 9 methyltransferases (G9a/HMTase1) and LSD1 $(69,100)$. The targets of CtBP repression include many of the tumor suppressor genes, and the ability to sense the intracellular NADH level makes CtBP a potential mediator of cell metabolic signaling and cancercell-specific transcriptional program (101-106). The interactions of CtBP with DNA-binding transcription factors are also regulated by the ratio of $\mathrm{NAD}^{+} / \mathrm{NADH}(97,104,107)$. Global increase of NADH was shown to improve the repression ability of CtBP via recruiting the repressive epigenetic modification enzymes. In other words, global increase of $\mathrm{NAD}^{+}$and $\mathrm{NADH}$ generates the same consequence in chromatin repression via distinct factors such as SIRTs and CtBP, respectively.

\section{GLOBAL VERSUS NUCLEAR DISTRIBUTION OF EERMS}

The nuclear membrane, unlike the mitochondria membrane, is most likely permeable by many small molecules including EERMs. Previous research rarely identified the known EERMs, which show uneven distribution in the cytoplasm and nucleus (108-111), suggesting the metabolic activities in the cytoplasm have a determinative influence on the enrichment of EERMs in the nucleus (Figure 2). That is why the

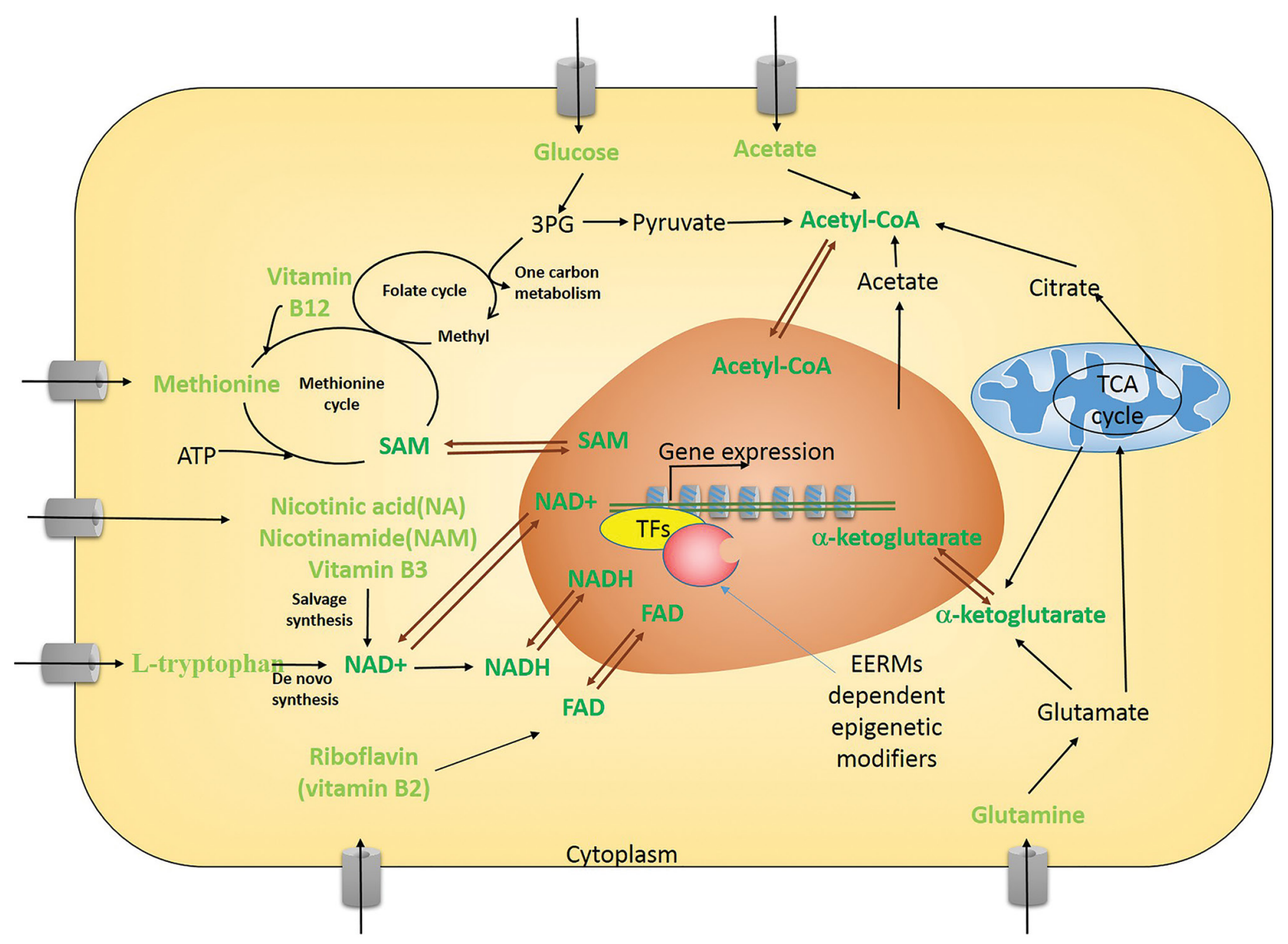

Figure 2. Global impact of EERMs on nuclear epigenetic events. The EERMs (dark green) including NAD ${ }^{+}, N A D H, S A M, A c e t y l-C o A$ and $\alpha-K G$ are mainly synthesized within cytoplasm and their cytoplasmic concentration is regulated through multiple metabolic pathways. These EERMs are freely permeabilized into the nucleus and act as cofactors for epigenetic modification enzymes. The metabolism precursors and co-enzymes (light green) that are necessary for synthesizing EERMs are absorbed mainly from extracellular supplies and their abundance may modulate the nuclear epigenetic activities via EERMs indirectly. 
cancer cell epigenome can be reshaped by consuming certain food components including vitamins and minerals $(112,113)$.

However, the number of metabolic enzymes presenting in the nucleus is still growing, making the nucleus a possible organelle with active metabolic activities (114). Thus it is likely that these nuclear-localized metabolic enzymes may produce EERMs in a much smaller scale within the nucleus and merely influence the nearby EERM-dependent epigenetic enzymes. Another possibility is that the localization of these metabolic enzymes in the nucleus is very brief and barely alters the EERM abundance in the nucleus temporarily. It is possible that these enzymes formed complexes with gene-specific transcription factors and directly participated in regulating gene expression. Several studies have provided compelling evidence that specific genes are regulated by EERMs, which are probably guided by transcription factors (Figure 3). For example, SAM synthases MAT2a and MAT2b were found to be nucleus-localized and were recruited to MafK-binding gene regulatory regions by forming a complex with MafK. The repression of MafK-targeted genes requires the interaction of MAT2 and MafK and the synthesis of SAM (26). MafK is not the only factor that binds to MAT2 in nucleus, many other chromatin-bound factors are found as interacting partners of MAT2, highly suggesting that a local supply of SAM is critical to genespecific chromatin modifications (25). In fact, the presence of metabolic enzymes in the nucleus is very common in many large scale screenings of chromatin-bound factors (115-117), suggesting local recruitment of these enzymes form important regulatory knots in regulating nuclear activities. For the nucleus-localized metabolic enzymes including ACL, nLDH, PDH, MAT2a, NMNAT-1 and others, most of these enzymes have not been proved to form complexes with gene-specific transcriptional factors to regulate

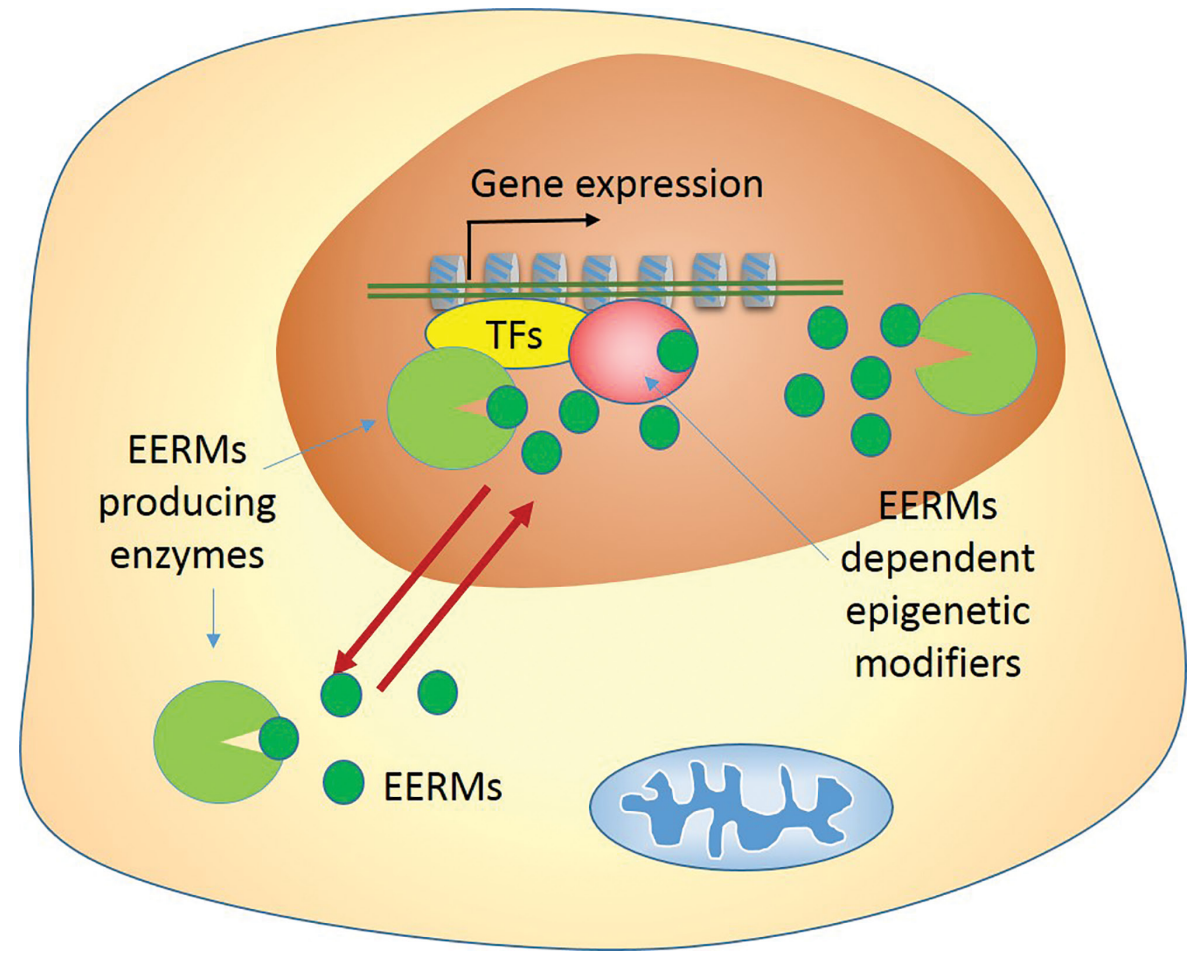

Figure 3. Local enrichment of EERMs and epigenetic modification. EERMs (green balls) can be produced in both the cytoplasm and nucleus, depending on the distribution of the EERM-producing enzymes. The EERM-producing enzymes localized in the nucleus may increase the enrichment of EERMs locally, facilitating the global regulation of epigenetic modifications by the EERM-dependent epigenetic modifiers. The EERM-producing enzymes may also form complexes with EERM-dependent epigenetic modifiers and/or gene-specific transcription factors and provide a local supply of EERMs to gene-specific regulation of epigenetic modifications.

epigenetic modifications except MAT2a. However, it is likely that these enzymes share a similar mechanism with MAT2a. In yeast, nuclear acetyl-CoA synthase 1 and 2 (Acs1 and Acs2) are required to supply acetyl-CoA locally to increase the acetylation of histones $(23,118)$, probably through gene-specific transcription factors. These preliminary observations ensure further studies on the sub-nucleus role of these related metabolites and respective enzymes.

\section{EERMS AS THERAPEUTIC TARGETS IN CANCER TREATMENT}

\section{Acetyl-CoA}

Nevertheless, EERMs are abundant metabolites and their status directly echoes intracellular metabolic activities.
Either directly or indirectly, some of the EERMs are known targets in many therapeutic applications in cancer treatment. For instance, acetyl-CoA is regulated through at least three mechanisms (Figure 2), and the dominant acetyl-CoA supply comes from glycolysis, as the majority of acetyl-CoA made from pyruvate stays inside of mitochondria. Therapeutic targeting of glycolysis may ablate the level of acetyl-CoA and have a significant impact on the acetylation of histones. More importantly, the shutdown of gene expression globally is associated with the reduction of acetyl-CoA (119-121). However, the benefit to cancer patients of glycolysis inhibition is still controversial, probably because the normal non-cancer cells are also under attack. 
In breast, liver, colon, lung and prostate cancers, cytoplasmic acetyl-CoA may be produced mainly through ACL-mediated citrate-to-acetyl-CoA conversion. Actually, ACL is abnormally presented in these cancer subtypes and correlated with the aggressiveness of tumors. The cancer cell specificity of this acetyl-CoA production pathway makes it an easier target in cancer therapy. Inhibition of ACL was found to alleviate some oncogenic gene expression through histone acetylation suppression (24).

The third pathway to produce acetyl-CoA has been identified recently and is through the direct ligation between acetate and CoA. So far, this pathway has only been identified in limited types of cancer including hepatocarcinoma and glioblastoma. However the enzyme ACSS2, which catalyzes the conversion of acetate to acetyl-CoA, was found to have increased expression in many human tumors (88). Thus, ACSS2 may be an alternate choice in reducing acetyl-CoA and histone acetylation, as well as gene expression.

\section{SAM}

The targeting of DNA methylation has received considerable attention, and several DNA methylation inhibitors have achieved significant success in killing cancer cells and reducing the tumor burden (122-124). Since SAM is a cofactor of DNMT, it is conceivable that targeting SAM can be another therapeutic opportunity. In fact, methionine dependence is a frequent phenomenon associated with breast, bladder, colon, glioma and kidney cancers owing to the deficiency of enzymes in the salvage methionine production pathway. Since the methionine cycle is important to produce SAM, this information further suggests SAM is a potential therapeutic target. Some studies, by knocking down or inhibiting MAT2s, actually show an apoptosis-induction effect in cancer cells $(125,126)$. However, most of the inhibitors of MAT2s lack specificity, which prevents their further application clinically.

\section{a-KG}

As we discussed earlier, competitive inhibition of $\alpha-\mathrm{KG}$ is frequently associated with tumor growth. The competitors of $\alpha-K G$ can be its structural analogs, such as succinate and fumarate, or its hydroxylated product 2-HG generated by mutant IDH. Some cancers accumulate succinate or fumarate owing to the deficiency in mitochondria and result in a pseudohypoxia status because succinate and fumarate compete with $\alpha-K G$ and result in the inhibition of PHD and the activation of HIFs. Extra fumarate and succinate also inhibit $\alpha-K G$-dependent demethylases and increase tumor cell growth. Succinate and fumarate accumulation can actually have a much more profound effect on the fate of cells (127). A proposed strategy is to apply cell-permeable $\alpha-K G$ derivatives to increase the intracellular $\alpha-K G$ abundance and to turn on the PHD and inactivate HIF (128). It is likely that the recovery of intracellular $\alpha-K G$ may decrease tumor cell growth. For instance, recent studies suggest IDH1 and IDH2 mutants can be targeted by compounds to reduce the production of $2-\mathrm{HG}$ $(129,130)$. Since $2-H G$ mainly competes with $\alpha-K G$, targeting IDH mutants favors $\alpha-K G$-mediated demethylation, which is proved to be the reason of cancer cell differentiation after treatment by these inhibitors. But another potential mechanism is the recovery of $\alpha-K G$ levels when IDH mutants are inhibited. In fact, even when there is no IDH mutation, some cancer cells also count on increased IDH activity to convert $\alpha-K G$ to citrate and lipid synthesis $(54,131)$, which are associated with decreased $\alpha-K G$ abundance.

\section{FAD}

FAD is known mainly as a cofactor of amine oxidase such as the histone demethylase LSD1. LSD1 overexpression is associated with several cancer cell lines and some types of tumors. It has become an attractive target for cancer therapy $(74,132)$. So a possible role of riboflavin, the precursor of FAD, in cancer was also tested in few studies, but the results are equivocal and the direct biochemical measurements of riboflavin in tumor tissue are actually quite rare. For example, increasing riboflavin reduces cancer risk significantly in colorectal cancer and cervical cancer models (133). The reason, as explained, may be the increased activity of MTHFR, another amine oxidase, and the rate-limiting enzyme to convert 5,10-methylene-THF to 5-methyl-THF. As we described, 5-methyl-THF is an important methyl group donor to form SAM, and DNA methylation and hypermethylation are preventive to cancer occurrence. However, whether this effect is solely through MTHFR is still in doubt, and the consequence of increased riboflavin is controversial. For example, weaker MTHFR activity owing to the allele polymorphism actually associates with a lower risk of cancer (43). Also, increasing riboflavin may promote LSD1 activity, which increases cancer risk. Therefore, further studies are required to clarify if riboflavin influences cancer risk or the therapeutic outcome.

\section{$\mathrm{NAD}^{+} / \mathrm{NADH}$}

In addition to its role as the carrier of protons in mitochondria, $\mathrm{NAD}^{+} / \mathrm{NADH}$ are also important substrates in some enzymatic activities catalyzed by PARPs, Sirtuins and CtBP, and all of these proteins are important regulators of the epigenetic status of chromatin. Cancer cells, when actively proliferating, have higher demands for $\mathrm{NAD}^{+} / \mathrm{NADH}$ biosynthesis. In fact, cancer cells have a higher amount of NADH and a greater turnover of NAD, making the cancer cells highly reduced in the intracellular environment. Therefore, the deficiency of vitamin B3, a precursor of $\mathrm{NAD}^{+}$synthesis, is known to be associated with an increased risk of cancer (134). Vitamin B3 deficiency has also been shown to increase the vulnerability of cancer cells to increased ROS, probably caused by the breakdown of the reduced intracellular status (135).

$\mathrm{NAD}^{+}$synthesis can be targeted by inhibiting NAMPT, the rate-limiting 
enzyme in the $\mathrm{NAD}^{+}$-synthesis pathway. NAMPT overexpression is known to be associated with multiple types of cancer (136). The NAMPT-specific inhibitory compound FK866 is shown to inhibit SIRT1 deacetylase activity and to result in the reprogramming of genomewide acetylation $(137,138)$. More importantly, FK866 is able to mimic the deficiency of vitamin B3 and to induce cancer-cell death via ROS-dependent apoptosis (139-141). Thus, $\mathrm{NAD}^{+}$-synthesis blocking may produce much more disastrous effects rather than influencing epigenetic modifications only (141).

NADH is more vulnerable to influence by intracellular metabolic activities as compared to the relative stable $\mathrm{NAD}^{+}$ level. For example, any fluctuation in energetic pathways, including glycolysis and the TCA cycle, will influence the abundance of NADH profoundly, which is consistent with the role of $\mathrm{NADH}$ as a proton carrier. Thus, any therapy targeting the glycolysis pathway reduces NADH abundance and influences the activity of co-repressor $\mathrm{CtBP}$, which may have a similar effect as HDAC inhibitors $(104,107)$.

\section{CONCLUSION}

Cell metabolism is a complicated network and is essential to providing cells the ability to synthesize thousands of intracellular, as well as extracellular, chemicals from the limited amount of substrate known to be essential nutrients for cell survival. Any fluctuation in these essential ingredients can have a profound impact on intracellular metabolic pathways (121). Sometimes, cells may develop novel routes, although perhaps less efficient, to overcome the challenges brought up by missing one or more essential substances, and they may synthesize the compounds that are needed to maintain cell life. The appearance of cancer cells can be the outcome of such adaptive changes of metabolic activities inside the cells. At least at the moment of emerging benign tumor cells, the adoption of abnormal metabolic activities may be the fundamental reason for cells gaining a growth advantage (142). Without DNA alteration, metabolic activities can influence gene expression through epigenetics, and such non-genetic variability can contribute to the somatic evolution of cancer cells.

However, current knowledge about cell metabolism is still far from complete (10). In addition to filling in the missing details of the pathways we have already identified, more challenges still exist which greatly limit our understanding of the correlation between cell metabolism and diseases, including cancer. The precise concentration of intracellular metabolites, including the EERMs described above, is hardly measurable without mentioning their compartmentation within cells. Some of the fluorescence probes have been developed to label important metabolites such as NADH, ATP and others (99), which are able to prove their uneven distribution within cells. But there is still a long way to go before we can accurately measure their change in distribution in the disease context. The transient and dynamic nature of metabolites further prohibits the investigation of their functions in each individual intracellular compartment. Conquering these challenges ensures further studies on the functions of these metabolites as cofactors of epigenetic enzymes beyond their role as metabolic products.

Moreover, the list of pairs of enzymes and metabolites that regulate the chromatin status is growing continuously and more novel interaction mechanisms between cell metabolism and nuclear activity are still under investigation. For instance, the lysine residuals of histone H3 were found to be crotonylated by a classical HAT p300 and this type of modification shows more potent activator function as compared to acetylation (143). Another example is the glycosylation of histones (144). In particular, the O-GlcNAc modification of histones was demonstrated recently to modulate gene transcription directly (145). Glycosylation is an extremely diversified process influenced by the glycosidic bond, the glycan structure and composition, as well as the glycan length, so the O-GlcNAc modification of histones may represent only one subtype of glycosylation and more diversified glycosylation of histones may also exist.

In addition, for complex diseases such as cancer, it is impossible to attribute one or two epigenetic events as major cause of any cancer. So the real challenge is how to systematically integrate both the variable metabolic activities and the global change of chromatin status by taking all the knowledge of interactions between EERMs and the corresponding epigenetic modifications into consideration. For example, a global increase of SAM does not increase genomewide DNA methylation, suggesting there are probably unknown mechanisms dominating the control of DNA methylation and reversing the SAM effect. Only with the full understanding of the mechanisms involved can the chromatin status be predicted based on the intracellular metabolism status.

Lastly, some studies illustrate that global changes of epigenetic modifications may correlate with the change of overall interphase chromatin compaction $(146,147)$. Moreover, decreased chromatin compaction increases the sensitivity of cells to chemotherapeutic reagents $(148,149)$. A direct application of such a correlation in clinics is to increase the sensitivity of cancer cells by subjecting the cells to chemotherapies composed of both chromatin-releasing agents and DNA-damage agents. Actually, metabolic manipulation can be a much easier way to decrease the compaction of chromatin if the impact of nutrient supply on chromatin compaction is predictable. For this reason, achieving a full understanding of the correlation between EERMs and chromatin status is very important in cancer therapies.

In conclusion, the coupling of cell metabolites with epigenetic modification enzymes ascertains the importance of epigenetic markers in verbalizing the cellular metabolic status. Cancer occurrence or cancer cell growth may take advantage of the metabolic alteration and label this 
information through epigenetics. With more emerging epidemiological evidence on the correlations between cancer occurrence and metabolic alterations in humans, EERMs may well bridge the gap in the understanding of the molecular mechanisms of such correlations. Further studies on EERMs and corresponding enzyme activities may also provide an opportunity to develop novel cancer treatment.

\section{ACKNOWLEDGMENTS}

This work is supported by the Science and Technology Development Fund (FDCT) of Macao SAR to LD (FDCT 025/2014/A1 \& FDCT 088/2014/A2), the Multi-Year Research Grant from the University of Macau to LD (MYRG201500037-FHS \& MYRG2015-00167-FHS) and the Marching Research Fund to LD (MRG022/DLJ/2015/FHS \& MRG023/ DLJ/2015/FHS).

\section{DISCLOSURE}

The authors declare that they have no competing interests as defined by Molecular Medicine, or other interests that might be perceived to influence the results and discussion reported in this paper.

\section{REFERENCES}

1. Henikoff S, Matzke MA. (1997) Exploring and explaining epigenetic effects. Trends Genet. 13:293-5.

2. Herceg Z, Vaissiere T. (2011) Epigenetic mechanisms and cancer: an interface between the environment and the genome. Epigenetics. 6:804-19.

3. Rossetto D, Avvakumov N, Cote J. (2012) Histone phosphorylation: a chromatin modification involved in diverse nuclear events. Epigenetics. 7:1098-108

4. Shiio Y, Eisenman RN. (2003) Histone sumoylation is associated with transcriptional repression. Proc. Natl. Acad. Sci. U. S. A. 100:13225-30.

5. Tan $\mathrm{M}$, et al. (2011) Identification of 67 histone marks and histone lysine crotonylation as a new type of histone modification. Cell. 146 1016-28.

6. Dehennaut V, Leprince D, Lefebvre T. (2014) O-glcnacylation, an epigenetic mark. Focus on the histone code, TET family proteins, and polycomb group proteins. Front Endocrinol. (Lausanne). 5:155.

7. Kothapalli $\mathrm{N}$, et al. (2005) Biological functions of biotinylated histones. J. Nutr. Biochem. 16:446-8.

8. Choudhuri S. (2011) From Waddington's epigenetic landscape to small noncoding RNA: some important milestones in the history of epigenetics research. Toxicol. Mech. Methods. 21:252-74.

9. Jaenisch R, Bird A. (2003) Epigenetic regulation of gene expression: how the genome integrates intrinsic and environmental signals. Nat. Genet. 33 Suppl:245-54.

10. Cantor JR, Sabatini DM. (2012) Cancer cell metabolism: one hallmark, many faces. Cancer Discov. 2:881-98.

11. Ward PS, Thompson CB. (2012) Metabolic reprogramming: a cancer hallmark even warburg did not anticipate. Cancer Cell. 21:297-308.

12. Jain M, et al. (2012) Metabolite profiling identifies a key role for glycine in rapid cancer cell proliferation. Science. 336:1040-4.

13. Donohoe DR, Bultman SJ. (2012) Metaboloepigenetics: interrelationships between energy metabolism and epigenetic control of gene expression. J. Cell. Physiol. 227:3169-77.

14. Flavahan WA, et al. (2016) Insulator dysfunction and oncogene activation in IDH mutant gliomas. Nature. 529:110-4.

15. Katada S, Imhof A, Sassone-Corsi P. (2012) Connecting threads: epigenetics and metabolism. Cell. 148 24-8.

16. Karpac J, Jasper H. (2011) Metabolic homeostasis: HDACs take center stage. Cell. 145:497-9.

17. Shyh-Chang N. et al. (2013) Influence of threonine metabolism on S-adenosylmethionine and histone methylation. Science. 339:222-6.

18. Shimazu T, et al. (2013) Suppression of oxidative stress by beta-hydroxybutyrate, an endogenous histone deacetylase inhibitor. Science. 339:211-4.

19. Li S, et al. (2015) Serine and SAM responsive complex SESAME regulates histone modification crosstalk by sensing cellular metabolism. Mol. Cell. 60:408-21.

20. Stover PJ. (2009) One-carbon metabolism-genome interactions in folate-associated pathologies. J. Nutrition. 139:2402-5.

21. Chang C, et al. (2015) AMPK-dependent phosphorylation of GAPDH triggers Sirt1 activation and is necessary for autophagy upon glucose starvation. Mol. Cell. 60:930-40.

22. Enzo E, et al. (2015) Aerobic glycolysis tunes YAP/TAZ transcriptional activity. EMBO J 34:1349-70.

23. Takahashi H, McCaffery JM, Irizarry RA, Boeke JD. (2006) Nucleocytosolic acetyl-coenzyme a synthetase is required for histone acetylation and global transcription. Mol. Cell. 23:207-17.

24. Wellen KE, Hatzivassiliou G, Sachdeva UM, Bui TV, Cross JR, Thompson CB. (2009) ATP-citrate lyase links cellular metabolism to histone acetylation. Science. 324:1076-80.

25. Igarashi K, Katoh Y. (2013) Metabolic aspects of epigenome: coupling of S-adenosylmethionine synthesis and gene regulation on chromatin by SAMIT module. Subcell. Biochem. 61:105-18.

26. Katoh Y, et al. (2011) Methionine adenosyltransferase II serves as a transcriptional corepressor of Maf oncoprotein. Mol. Cell. 41:554-66.
27. Yang W, et al. (2011) Nuclear PKM2 regulates beta-catenin transactivation upon EGFR activation. Nature. 480:118-22.

28. Yang W, et al. (2012) PKM2 phosphorylates histone $\mathrm{H} 3$ and promotes gene transcription and tumorigenesis. Cell. 150:685-96.

29. Boyes J, Bird A. (1991) DNA methylation inhibits transcription indirectly via a methyl-CpG binding protein. Cell. 64:1123-34.

30. Kulis M, Esteller M. (2010) DNA methylation and cancer. Adv. Genet. 70:27-56.

31. Esteller M. (2002) CpG island hypermethylation and tumor suppressor genes: a booming present, a brighter future. Oncogene. 21:5427-40.

32. Baylin SB. (2005) DNA methylation and gene silencing in cancer. Nat. Clin. Pract. Oncol. 2 Suppl 1:S4-11.

33. Robertson KD. (2005) DNA methylation and human disease. Nat. Rev. Genet. 6:597-610.

34. Boyes J, Bird A. (1992) Repression of genes by DNA methylation depends on CpG density and promoter strength: evidence for involvement of a methyl-CpG binding protein. EMBO J. 11:327-33.

35. Martin C, Zhang Y. (2005) The diverse functions of histone lysine methylation. Nat. Rev. Mol. Cell Biol. 6:838-49.

36. Volkel P, Angrand PO. (2007) The control of histone lysine methylation in epigenetic regulation. Biochimie. 89:1-20.

37. Cloutier P, Lavallee-Adam M, Faubert D, Blanchette M, Coulombe B. (2013) A newly uncovered group of distantly related lysine methyltransferases preferentially interact with molecular chaperones to regulate their activity. PLoS Genet. 9:e1003210.

38. Feil R, Fraga MF. (2011) Epigenetics and the environment: emerging patterns and implications. Nat. Rev. Genet. 13:97-109.

39. Shafqat N, et al. (2013) Insight into S-adenosylmethionine biosynthesis from the crystal structures of the human methionine adenosyltransferase catalytic and regulatory subunits. Biochem. J. 452:27-36.

40. Wu G. (2009) Amino acids: metabolism, functions, and nutrition. Amino Acids. 37:1-17.

41. Kulkarni A, Dangat K, Kale A, Sable P, ChavanGautam P, Joshi S. (2011) Effects of altered maternal folic acid, vitamin B12 and docosahexaenoic acid on placental global DNA methylation patterns in Wistar rats. PloS One. 6:e17706.

42. Locasale JW. (2013) Serine, glycine and onecarbon units: cancer metabolism in full circle. Nat. Rev. Cancer. 13:572-83.

43. Kimura M, Umegaki K, Higuchi M, Thomas P, Fenech M. (2004) Methylenetetrahydrofolate reductase C677T polymorphism, folic acid and riboflavin are important determinants of genome stability in cultured human lymphocytes. J. Nutr. 134:48-56.

44. Wallace DC. (2012) Mitochondria and cancer. Nat. Rev. Cancer. 12:685-98.

45. Bergstrom M, et al. (1987) PET study of methionine accumulation in glioma and normal brain tissue: competition with branched chain amino acids. J. Comput. Assist. Tomogr. 11:208-13. 
46. Judde JG, Ellis M, Frost P. (1989) Biochemical analysis of the role of transmethylation in the methionine dependence of tumor cells. Cancer Res. 49:4859-65.

47. Locasale JW, et al. (2011) Phosphoglycerate dehydrogenase diverts glycolytic flux and contributes to oncogenesis. Nat. Genet. 43:869-74.

48. Maddocks OD, Labuschagne CF, Adams PD, Vousden KH. (2016) Serine metabolism supports the methionine cycle and DNA/RNA methylation through de novo ATP synthesis in cancer cells. Mol. Cell. 61:210-21.

49. Luka Z, Mudd SH, Wagner C. (2009) Glycine $\mathrm{N}$-methyltransferase and regulation of S-adenosylmethionine levels. J. Biol. Chem. 284:22507-11.

50. Shen H, Laird PW. (2013) Interplay between the cancer genome and epigenome. Cell. 153:38-55.

51. Yang HB, et al. (2015) Acetylation of MAT IIalpha represses tumour cell growth and is decreased in human hepatocellular cancer. Nat. Commun. 6:6973.

52. Wang P, et al. (2009) Global analysis of H3K4 methylation defines MLL family member targets and points to a role for MLL1-mediated H3K4 methylation in the regulation of transcriptional initiation by RNA polymerase II. Mol. Cell. Biol. 29:6074-85.

53. Poore B, Siegel N, Park JK, Hwang BJ, IAfif I, Le A. (2015) Glucose-independent glutamine-driven TCA cycle in cancer cells. In: Glutamine in Clinical Nutrition. Springer, New York, pp. 77-85.

54. Mullen AR, et al. (2014) Oxidation of alphaketoglutarate is required for reductive carboxylation in cancer cells with mitochondrial defects. Cell Rep. 7:1679-90.

55. Loenarz C, Schofield CJ. (2011) Physiological and biochemical aspects of hydroxylations and demethylations catalyzed by human 2-oxoglutarate oxygenases. Trends Biochem. Sci. 36:7-18.

56. Ito S, et al. (2011) Tet proteins can convert 5-methylcytosine to 5-formylcytosine and 5-carboxylcytosine. Science. 333:1300-3.

57. Tahiliani M, et al. (2009) Conversion of 5-methylcytosine to 5-hydroxymethylcytosine in mammalian DNA by MLL partner TET1. Science. 324:930-5.

58. Kohli RM, Zhang Y. (2013) TET enzymes, TDG and the dynamics of DNA demethylation. Nature. 502:472-9.

59. Kooistra SM, Helin K. (2012) Molecular mechanisms and potential functions of histone demethylases. Nat. Rev. Mol. Cell. Biol. 13:297-311.

60. W. Xu, et al. (2011) Oncometabolite 2-hydroxyglutarate is a competitive inhibitor of alphaketoglutarate-dependent dioxygenases. Cancer Cell. 19:17-30.

61. Figueroa ME, et al. (2010) Leukemic IDH1 and IDH2 mutations result in a hypermethylation phenotype, disrupt TET2 function, and impair hematopoietic differentiation. Cancer Cell. 18:553-67.
62. Fendt SM, et al. (2013) Reductive glutamine metabolism is a function of the alpha-ketoglutarate to citrate ratio in cells. Nat. Commun. 4:2236.

63. Witte T, Plass C, Gerhauser C. (2014) Pan-cancer patterns of DNA methylation. Genome Med. 6:66

64. Weisenberger DJ. (2014) Characterizing DNA methylation alterations from The Cancer Genome Atlas. J. Clin. Invest. 124:17-23.

65. Keenan MM, et al. (2015) ACLY and ACC1 Regulate Hypoxia-Induced Apoptosis by Modulating ETV4 via alpha-ketoglutarate. PLoS. Genet. 11:e1005599.

66. Xiao M, et al. (2012) Inhibition of alpha-KGdependent histone and DNA demethylases by fumarate and succinate that are accumulated in mutations of FH and SDH tumor suppressors. Genes Dev. 26:1326-38.

67. Terunuma A, et al. (2014) MYC-driven accumulation of 2-hydroxyglutarate is associated with breast cancer prognosis. J. Clin. Invest. 124:398-412.

68. Shim EH, et al. (2014) 1-2-Hydroxyglutarate: An epigenetic modifier and putative oncometabolite in renal cancer. Cancer Discov. 4:1290-8.

69. Shi Y, et al. (2004) Histone demethylation mediated by the nuclear amine oxidase homolog LSD1. Cell. 119:941-53.

70. Anand R, Marmorstein R. (2007) Structure and mechanism of lysine-specific demethylase enzymes. J. Biol. Chem. 282:35425-9.

71. Metzger E, et al. (2005) LSD1 demethylates repressive histone marks to promote androgen-receptordependent transcription. Nature. 437:436-9.

72. Schulte JH, et al. (2009) Lysine-specific demethylase 1 is strongly expressed in poorly differentiated neuroblastoma: implications for therapy. Cancer Res. 69:2065-71.

73. Wang Y, et al. (2009) LSD1 is a subunit of the NuRD complex and targets the metastasis programs in breast cancer. Cell. 138:660-72.

74. Hayami S, et al. (2011) Overexpression of LSD1 contributes to human carcinogenesis through chromatin regulation in various cancers. Int. J. Cancer. 128:574-86.

75. Teperino R, Schoonjans K, Auwerx J. (2010) Histone methyl transferases and demethylases; can they link metabolism and transcription? Cell Metab. 12:321-7.

76. Liu D, Zempleni J. (2014) Low activity of LSD1 elicits a pro-inflammatory gene expression profile in riboflavin-deficient human T Lymphoma Jurkat cells. Genes Nutr. 9:422.

77. Liu D, Zempleni J. (2014) Transcriptional regulation of the albumin gene depends on the removal of histone methylation marks by the FAD-dependent monoamine oxidase lysine-specific demethylase 1 in HepG2 human hepatocarcinoma cells. J. Nutr. 144:997-1001

78. Hino S, et al. (2012) FAD-dependent lysine-specific demethylase-1 regulates cellular energy expenditure. Nat. Commun. 3:758.

79. Giancaspero TA, et al. (2013) FAD synthesis and degradation in the nucleus create a local flavin cofactor pool. J. Biol. Chem. 288:29069-80.
80. Marmorstein R, Zhou MM. (2014) Writers and readers of histone acetylation: structure, mechanism, and inhibition. Cold Spring Harb. Perspect. Biol. 6:a018762.

81. Galdieri L, Vancura A. (2012) Acetyl-CoA carboxylase regulates global histone acetylation. J. Biol. Chem. 287:23865-76.

82. Carracedo A, Cantley LC, Pandolfi PP. (2013) Cancer metabolism: fatty acid oxidation in the limelight. Nat. Rev. Cancer. 13:227-32.

83. Sutendra G, et al. (2014) A nuclear pyruvate dehydrogenase complex is important for the generation of acetyl-CoA and histone acetylation. Cell. 158:84-97.

84. Yoshii Y, Furukawa T, Saga T, Fujibayashi Y (2015) Acetate/acetyl-CoA metabolism associated with cancer fatty acid synthesis: overview and application. Cancer Lett. 356:211-6.

85. Shyh-Chang N, Daley GQ. (2015) Metabolic switches linked to pluripotency and embryonic stem cell differentiation. Cell Metab. 21:349-50.

86. Schug ZT, et al. (2015) Acetyl-CoA synthetase 2 promotes acetate utilization and maintains cancer cell growth under metabolic stress. Cancer Cell. 27:57-71.

87. Mashimo T. et al. (2014) Acetate is a bioenergetic substrate for human glioblastoma and brain metastases. Cell. 159:1603-14.

88. Comerford SA, et al. (2014) Acetate dependence of tumors. Cell. 159:1591-602.

89. Roth M, Chen WY. (2014) Sorting out functions of sirtuins in cancer. Oncogene. 33:1609-20.

90. Nikiforov A, Kulikova V, Ziegler M. (2015) The human NAD metabolome: Functions, metabolism and compartmentalization. Crit. Rev. Biochem. Mol. Biol. 50:284-97.

91. Schweiger M, et al. (2001) Characterization of recombinant human nicotinamide mononucleotide adenylyl transferase (NMNAT), a nuclear enzyme essential for NAD synthesis. FEBS Lett. 492:95-100.

92. Wang J, et al. (2011) Toward an understanding of the protein interaction network of the human liver. Mol. Syst. Biol. 7:536.

93. Castonguay Z, Auger C, Thomas SC, Chahma M, Appanna VD. (2014) Nuclear lactate dehydrogenase modulates histone modification in human hepatocytes. Biochem. Biophys. Res. Commun. 454:172-7.

94. Zhang T, Kraus WL. (2010) SIRT1-dependent regulation of chromatin and transcription: linking $\mathrm{NAD}(+)$ metabolism and signaling to the control of cellular functions. Biochim Biophys Acta. 1804:1666-75.

95. Kraus WL. (2008) Transcriptional control by PARP-1: chromatin modulation, enhancerbinding, coregulation, and insulation. Curr. Opin. Cell. Biol. 20:294-302.

96. Wright BK, et al. (2012) NADH distribution in live progenitor stem cells by phasor-fluorescence lifetime image microscopy. Biophys. J. 103:L7-9.

97. Zhang Q, Piston DW, Goodman RH. (2002) Regulation of corepressor function by nuclear NADH. Science. 295:1895-97. 
98. Araki T, Sasaki Y, Milbrandt J. (2004) Increased nuclear NAD biosynthesis and SIRT1 activation prevent axonal degeneration. Science. 305:1010-3.

99. Zhao Y, Yang Y. (2015) Profiling metabolic states with genetically encoded fluorescent biosensors for NADH. Curr. Opin. Biotechnol. 31:86-92.

100. Chinnadurai G. (2002) CtBP, an unconventional transcriptional corepressor in development and oncogenesis. Mol. Cell. 9:213-24.

101. Wang L, Zhou H, Wang Y, Cui G, Di LJ. (2015) CtBP maintains cancer cell growth and metabolic homeostasis via regulating SIRT4. Cell Death Dis. 6:e1620.

102. Madison DL, Lundblad JR. (2010) C-terminal binding protein and poly(ADP)ribose polymerase 1 contribute to repression of the p21(waf1/cip1) promoter. Oncogene. 29:6027-39.

103. Deng Y, et al. (2012) Transcriptional downregulation of Brca1 and E-cadherin by CtBP1 in breast cancer. Mol. Carcinog. 51:500-7.

104. Di LG, Fernandez AG, De Siervi A, Longo DL, Gardner K. (2010) Transcriptional regulation of BRCA1 expression by a metabolic switch. Nat. Struct. Mol. Biol. 17:1406-13.

105. Paliwal S, Ho N, Parker D, Grossman SR. (2012) CtBP2 Promotes Human Cancer Cell Migration by Transcriptional Activation of Tiam1. Genes Cancer. 3:481-90.

106. Paliwal S, Kovi RC, Nath B, Chen YW, Lewis BC, Grossman SR. (2007) The alternative reading frame tumor suppressor antagonizes hypoxia-induced cancer cell migration via interaction with the $\mathrm{COOH}$-terminal binding protein corepressor. Cancer Res. 67:9322-9.

107. Di LJ, et al. (2013) Genome-wide profiles of CtBP link metabolism with genome stability and epithelial reprogramming in breast cancer. Nat. Commun. 4:1449.

108. Houtkooper RH, Canto C, Wanders RJ, Auwerx J. (2010) The secret life of NAD+: an old metabolite controlling new metabolic signaling pathways. Endocr. Rev. 31:194-223.

109. Stein LR, Imai S. (2012) The dynamic regulation of NAD metabolism in mitochondria. Trends Endocrinol. Metab. 23:420-8.

110. Talcott B, Moore MS. (1999) Getting across the nuclear pore complex. Trends Cell. Biol. 9:312-8.

111. Wente SR, Rout MP. (2010) The nuclear pore complex and nuclear transport. Cold Spring Harb. Perspect. Biol. 2 a000562.

112. Milner JA. (2008) Nutrition and cancer: essential elements for a roadmap. Cancer Lett. 269:189-98.

113. Supic G, Jagodic M, Magic Z. (2013) Epigenetics: a new link between nutrition and cancer. Nutr. Cancer. 65 781-92.

114. Zecchin A, Stapor PC, Goveia J, Carmeliet P. (2015) Metabolic pathway compartmentalization: an underappreciated opportunity? Curr. Opin. Biotechnol. 34:73-81.

115. Mittler G, Butter F, Mann M. (2009) A SILACbased DNA protein interaction screen that identifies candidate binding proteins to functional DNA elements. Genome Res. 19:284-93.
116. Ho SW, Jona G, Chen CT, Johnston M, Snyder M. (2006) Linking DNA-binding proteins to their recognition sequences by using protein microarrays. Proc. Natl. Acad. Sci. U. S. A. 103:9940-5.

117. Hall DA, Zhu H, Zhu X, Royce T, Gerstein M, Snyder M. (2004) Regulation of gene expression by a metabolic enzyme. Science. 306:482-4.

118. Falcon AA, Chen S, Wood MS, Aris JP. (2010) Acetyl-coenzyme A synthetase 2 is a nuclear protein required for replicative longevity in Saccharomyces cerevisiae. Mol. Cell. Biochem. 333:99-108

119. Moussaieff A, et al. (2015) Glycolysis-mediated changes in acetyl-CoA and histone acetylation control the early differentiation of embryonic stem cells. Cell. Metab. 21:392-402.

120. Cluntun AA, Huang H, Dai L, Liu X, Zhao Y, Locasale JW. (2015) The rate of glycolysis quantitatively mediates specific histone acetylation sites. Cancer Metab. 3:10.

121. Liu XS, Little JB, Yuan ZM. (2015) Glycolytic metabolism influences global chromatin structure. Oncotarget. 6:4214-25.

122. Issa JP. (2005) Optimizing therapy with methylation inhibitors in myelodysplastic syndromes: dose, duration, and patient selection. Nat. Clin. Pract. Oncol. 2 Suppl 1:S24-9.

123. Chiappinelli KB, et al. (2015) Inhibiting DNA methylation causes an interferon response in cancer via dsRNA including endogenous retroviruses. Cell. 162:974-86.

124. Matei D, et al. (2012) Epigenetic resensitization to platinum in ovarian cancer. Cancer Res. 72:2197-205.

125. Wang YC, Chiang EP. (2012) Low-dose methotrexate inhibits methionine S-adenosyltransferase in vitro and in vivo. Mol. Med. 18:423-32.

126. Wang Q, Liu QY, Liu ZS, Qian Q, Sun Q, Pan DY. (2008) Inhibition of hepatocelluar carcinoma MAT2A and MAT2beta gene expressions by single and dual small interfering RNA. J. Exp. Clin. Cancer Res. 27:72

127. Aspuria PJ, et al. (2014) Succinate dehydrogenase inhibition leads to epithelial-mesenchymal transition and reprogrammed carbon metabolism. Cancer Metab. 2:21.

128. MacKenzie ED, et al. (2007) Cell-permeating alpha-ketoglutarate derivatives alleviate pseudohypoxia in succinate dehydrogenasedeficient cells. Mol. Cell. Biol. 27:3282-9.

129. Rohle D, et al. (2013) An inhibitor of mutant IDH1 delays growth and promotes differentiation of glioma cells. Science. 340:626-30.

130. Wang F, et al. (2013) Targeted inhibition of mutant IDH2 in leukemia cells induces cellular differentiation. Science. 340 622-626.

131. Wise DR, et al. (2011) Hypoxia promotes isocitrate dehydrogenase-dependent carboxylation of alpha-ketoglutarate to citrate to support cell growth and viability. Proc. Natl. Acad. Sci. U. S. A. 108:19611-6.

132. Lynch JT, Harris WJ, Somervaille TC. (2012) LSD1 inhibition: a therapeutic strategy in cancer? Expert Opin. Ther. Targets. 16:1239-49.
133. Powers HJ. (2005) Interaction among folate, riboflavin, genotype, and cancer, with reference to colorectal and cervical cancer. J. Nutr. 135:2960S-6S.

134. Kirkland JB. (2003) Niacin and carcinogenesis. Nutr. Cancer. 46:110-8.

135. Pollak N, Dolle C, Ziegler M. (2007) The power to reduce: pyridine nucleotides - small molecules with a multitude of functions. Biochem. J. 402:205-18.

136. Garten A, Petzold S, Korner A, Imai S, Kiess W. (2009) Nampt: linking NAD biology, metabolism and cancer. Trends Endocrinol. Metab. 20:130-8.

137. Liu TF, Yoza BK, El Gazzar M, Vachharajani VT, McCall CE. (2011) NAD+-dependent SIRT1 deacetylase participates in epigenetic reprogramming during endotoxin tolerance. J. Biol. Chem. 286:9856-64.

138. Song TY, Yeh SL, Hu ML, Chen MY, Yang NC. (2015) A Nampt inhibitor FK866 mimics vitamin B3 deficiency by causing senescence of human fibroblastic Hs68 cells via attenuation of NAD(+)-SIRT1 signaling. Biogerontology. 16:789-800.

138. Moore Z, et al. (2015) NAMPT inhibition sensitizes pancreatic adenocarcinoma cells to tumor-selective, PAR-independent metabolic catastrophe and cell death induced by betalapachone. Cell Death Dis. 6:e1599.

140 Gehrke I, et al. (2014) On-target effect of FK866, a nicotinamide phosphoribosyl transferase inhibitor, by apoptosis-mediated death in chronic lymphocytic leukemia cells. Clin. Cancer Res. 20:4861-72.

141. Chini CC, et al. (2014) Targeting of NAD metabolism in pancreatic cancer cells: potential novel therapy for pancreatic tumors. Clin. Cancer Res. 20:120-30.

142. Brock A, Chang H, Huang S. (2009) Non-genetic heterogeneity - a mutation-independent driving force for the somatic evolution of tumours. Nat. Rev. Genet. 10:336-42.

143. Sabari BR, et al. (2015) Intracellular crotonyl-CoA stimulates transcription through p300-catalyzed histone crotonylation. Mol. Cell. 58:203-15.

144. Nagel AK, Ball LE. (2015) Intracellular protein O-GlcNAc modification integrates nutrient status with transcriptional and metabolic regulation. Adv. Cancer Res. 126:137-66.

145. Sakabe K, Wang Z, Hart GW. (2010) Beta-Nacetylglucosamine (O-GlcNAc) is part of the histone code. Proc. Natl. Acad. Sci. U. S. A. 107:19915-20.

146. Ito T. (2007) Role of histone modification in chromatin dynamics. J. Biochem. 141:609-14

147. Campos EI, Reinberg D. (2009) Histones: annotating chromatin. Annu. Rev. Genet. 43:559-99.

148. Takata H, et al. (2013) Chromatin compaction protects genomic DNA from radiation damage. PLoS One. 8:e75622.

149. Falk M, Lukasova E, Kozubek S. (2008) Chromatin structure influences the sensitivity of DNA to gamma-radiation. Biochim. Biophys. Acta. 1783:2398-414.

Cite this article as: Zhao Z, Wang L, Di L-J. (2016)

Compartmentation of metabolites in regulating epigenomes of cancer. Mol. Med. 22:349-60. 